\title{
The association analysis of IncRNA HOTAIR genetic variants and gastric cancer risk in a Chinese population
}

\author{
Mulong Du ${ }^{1, *}$, Weizhi Wang ${ }^{2, *}$, Hua Jin ${ }^{3, *}$, Qiaoyan Wang ${ }^{1}$, Yuqiu Ge $^{1}$, Jiafei Lu ${ }^{1}$, \\ Gaoxiang Ma ${ }^{1}$, Haiyan $\mathrm{Chu}^{1,4}{ }^{1}$, Na Tong ${ }^{1,4}$, Haixia Zhu ${ }^{3}$, Meilin Wang ${ }^{1,4}$, Fulin Qiang ${ }^{3}$, \\ Zhengdong Zhang ${ }^{1,4}$ \\ ${ }^{1}$ Department of Environmental Genomics, Jiangsu Key Laboratory of Cancer Biomarkers, Prevention and Treatment, Cancer \\ Center, Nanjing Medical University, Nanjing, China \\ ${ }^{2}$ Department of General Surgery, the First Affiliated Hospital of Nanjing Medical University, Nanjing, China \\ ${ }^{3}$ Core Laboratory, Nantong Tumor Hospital, Nantong, China \\ ${ }^{4}$ Department of Genetic Toxicology, the Key Laboratory of Modern Toxicology of Ministry of Education, School of Public \\ Health, Nanjing Medical University, Nanjing, China \\ "These authors have contributed equally to this work
}

Correspondence to:

Zhengdong Zhang, e-mail: drzdzhang@gmail.com

Keywords: long noncoding RNA, HOTAIR, genetic variants, gastric cancer

Received: May 12, $2015 \quad$ Accepted: August 24, $2015 \quad$ Published: september 04, 2015

\section{ABSTRACT}

The HOX transcript antisense intergenic RNA (HOTAIR), a well-known long noncoding RNA, is involved in pathogenesis and progress of multiple tumors. Its ectopic expression and biological functions have been observed in gastric cancer. In this study, we conducted a two-stage case-control study to evaluate whether genetic variations of HOTAIR were associated with gastric cancer risk. We identified that a single nucleotide polymorphism (SNP) rs4759314 was significantly associated with the increased gastric cancer risk with an odds ratio (OR) of 1.39 [95\% confidence interval $(\mathrm{CI})=1.13-1.71, P=0.002]$ in the combined sets. Further functional experiments revealed the allele-specific effects on HOTAIR and HOXC11 expressions in gastric cancer tissues, of which HOTAIR and HOXC11 expressions of individuals carrying with AG genotype were much higher than those with AA genotype; similarly, the effects occurred in intronic promoter activities, of which the promoter activity of $\mathbf{G}$ allele was more pronounced than that of $A$ allele. Interestingly, we identified a novel potential oncogene HOXC11 in gastric cancer pathogenesis with differential expression in gastric cancer tissues by association analysis with candidate gene strategy. These results suggest that SNP rs4759314 of HOTAIR acts as a potential biomarker for predicting gastric cancer, and the role of HOXC11 in gastric cancer etiology is warranted to further investigation.

\section{INTRODUCTION}

Gastric cancer (GC) is one of the most common malignant tumors with high mortality in the world. Recently, the GLOBOCAN project (http://globocan. iarc.fr) has reported that the worldwide morbidity of $\mathrm{GC}$ is the fifth, and the third for mortality [1]. Although a slight decline in GC incidence and mortality [2], new diagnosed cases and the death each year are still a huge number in Asian countries, predominantly in China, where specifically GC is the third fatal cause, following lung cancer and liver cancer [1]. However, little is known about the exactly mechanism of GC development and progress, despite that accumulating evidences indicate the significant association between environmental factors and epigenetic/genetic effects and GC etiology.

To data, long non-coding RNAs (lncRNAs) have been extremely gained attention for their wide range of biological regulatory functions [3]. Many studies have demonstrated that lncRNAs may be involved in pathogenesis of cancers, referring to the levels of transcription, post-transcription and epigenome [4-6]. 
Especially, lncRNA HOX transcript antisense intergenic RNA (HOTAIR) was earlier identified participated in the development and progression of malignancies $[4,7,8]$. Gupta et al. initially indicated that HOTAIR expression was up-expressed in breast cancer tissues, as well as be related to breast cancer progression [4]; and further studies showed that HOTAIR could induce genome-wide retargeting of Polycomb repressive complex 2 (PRC2), contributing to altered $\mathrm{H} 3$ lysine 27 methylation $[4,9]$. In $\mathrm{GC}$, the HOTAIR level was highly expressed in tumors and exerted strong association with clinical phenotypes, such as venous invasion, lymph node metastases and survival [10, 11]; besides, HOTAIR could sponge miR-331-3p, functioning as a competing endogenous RNA, to regulate HER2 expression in GC cells [12].

Recently, considerable studies have investigated the effects of lncRNAs genetic variations (majorly composed of single nucleotide polymorphisms, SNPs) on cancer susceptibility. The tagging SNP (tagSNP) rs2839698 of lncRNA $H 19$ firstly identified was significantly associated with the decreased bladder cancer risk [13], as well that SNP rs6434568 in IncRNA PCGEM1 was related to prostate cancer risk [14]. In our previous study, we observed a protective effect of SNP rs7958904 in HOTAIR on colorectal cancer susceptibility and an allele-specific cellular phenotype in colorectal cancer proliferation [15]. Furthermore, although SNP rs12826786 of HOTAIR was related to gastric cardia adenocarcinoma risk, little evidence could support the genetic effects of HOTAIR SNPs on GC susceptibility and gene functions. Thus, in this study, we conducted a two-stage case-control study to evaluate the association between HOTAIR SNPs and $\mathrm{GC}$ risk in a Chinese population, and assess the biological effect of the SNPs.

\section{RESULTS}

\section{Characteristics of study subjects}

In this study, a total of $1275 \mathrm{GC}$ cases and 1646 controls were recruited in two stages, and there were no significant differences between GC cases and cancerfree controls for each stage regarding to age and sex (all $P>0.05$, Supplementary Table 1). Furthermore, in the combined set, $61.3 \%$ of GC cases were in non-cardia type, and $33.6 \%$ in the cardia and $5.1 \%$ in the both; and a slightly higher percentage was in diffuse type than those in the intestinal ( $54.4 \%$ and $45.6 \%$, respectively). Besides, $23.1 \%, 24.6 \%, 35.5 \%$, and $16.8 \%$ of GC cases were in TNM stage I, II, III, and IV, respectively.

\section{Associations of tagSNPs and GC risk}

In the test set, the genotypes distributions of 3 SNPs (i.e. rs4759314, rs7958904 and rs874945) among the controls were in accordance with Hardy-Weinberg equilibrium ( $P=0.699,0.271$ and 0.672 , respectively; Supplementary Table 2). Subsequently, four genetic effect models were performed to assess the associations of candidate SNPs and GC risk. We found that only SNP rs4759314 was associated with GC risk (Table 1): although the genotypes frequency distributions of SNP rs4759314 in an additive genetic model was of marginal differences between cases and controls $\left(P_{\text {trend }}=0.049\right)$, its genetic effect in dominant model significantly associated with $\mathrm{GC}$ risk $(P=0.030 ; \mathrm{OR}=1.34,95 \% \mathrm{CI}=1.03-1.73)$, especially the more risk was found in the heterozygotes $(\mathrm{OR}=1.36,95 \% \mathrm{CI}=1.05-1.77)$ rather than in the minor homozygotes $(\mathrm{OR}=0.75,95 \% \mathrm{CI}=0.19-2.99)$. No significant association with GC risk was observed in both SNPs rs7958904 and rs874945.

Subsequently, we performed an independent validation set and identified that rs4759314 still had significant effects on gastric cancer risk (Table 1). Compared with the AA genotypes, more individuals with $\mathrm{G}$ allele were genotyped in cases than that in controls $(P=0.004, \mathrm{OR}=1.99,95 \% \mathrm{CI}=1.31-3.04$ in the dominant model). Similarly, the effect of heterozygotes in co-dominant model was more significant than that of homozygotes ( $P=0.002$ for heterozygote and $P=0.506$ for homozygote). When combined these two stages, the AG/GG genotypes were also dramatically associated with $\mathrm{GC}$ risk $(\mathrm{OR}=1.45,95 \% \mathrm{CI}=1.17-1.80, P<0.001)$, and the $\mathrm{AG}$ genotype was particular in main effect $(\mathrm{OR}=1.47$, $95 \% \mathrm{CI}=1.18-1.84, P<0.001)$.

In addition, stratified analyses of SNP rs4759314 were performed by demographic and clinical characteristics. We identified more prominent risk effect of SNP rs4759314 AG/GG genotypes in subgroup of the older subjects (age $>63, P<0.001)$ and males $(P<0.001)$ (Figure 1), and subsequently found that the AG/GG genotypes were significantly associated with $\mathrm{GC}$ risk in each subgroup of tumor site, histological type and TNM stage (Table 2).

\section{The relationship of SNP rs4759314 on HOTAIR and $\mathrm{HOXC11}$ expression level}

We next investigated whether the GC susceptibility SNP rs4759314 has an allele-specific effect on nearby genes. Supplementary Figure 1 presents the loci of SNP rs4759314 and its nearby genes, thus we measured the expression level of its host gene HOTAIR and nearby gene HOXC11 in $63 \mathrm{GC}$ tissues by using real-time RT-PCR. As shown in Figure 2a-2b, individuals with the AG genotype expressed higher HOTAIR and HOXC11 levels than those with AA genotype in GC tissues $(P=0.032$ for HOTAIR and $P=0.036$ for $H O X C 11)$. In addition, we explored The Cancer Genome Atlas (TCGA) database to assess the effect of SNP rs4759314 on HOXC11 expression level. 
Table 1: The association between SNP rs4759314 of HOTAIR and gastric cancer susceptibility in two-stage case-control study

\begin{tabular}{|c|c|c|c|c|c|c|c|c|}
\hline \multirow{3}{*}{ SNP } & \multirow{3}{*}{ Stages } & \multirow{3}{*}{ Cases $^{b}$} & \multirow{3}{*}{ Controls $^{b}$} & \multirow{3}{*}{$\begin{array}{c}\text { MAF } \\
\text { Case/ } \\
\text { Control }\end{array}$} & \multicolumn{3}{|c|}{ OR $(95 \% \text { CI })^{d}$} & \multirow{3}{*}{$\begin{array}{c}\qquad P^{\mathrm{d}} \\
\text { Additive }\end{array}$} \\
\hline & & & & & \multirow{2}{*}{$\begin{array}{l}\text { Additive/dominant/ } \\
\text { recessive model }\end{array}$} & \multicolumn{2}{|c|}{ Co-dominant model } & \\
\hline & & & & & & het $^{\mathrm{e}}$ & hom $^{\mathrm{e}}$ & \\
\hline rs4759314 & Test set & $624 / 126 / 3$ & $915 / 136 / 6$ & $0.087 / 0.070$ & $\begin{array}{c}1.29(1.00-1.65) / \\
1.34(1.03-1.73) / \\
0.71(0.18-2.86)\end{array}$ & $\begin{array}{c}1.36 \\
(1.05-1.77)\end{array}$ & $\begin{array}{c}0.75 \\
(0.19-2.99)\end{array}$ & 0.047 \\
\hline \multirow[t]{2}{*}{$\mathrm{A} / \mathrm{G}^{\mathrm{a}}$} & $\begin{array}{l}\text { Validation } \\
\text { set }\end{array}$ & $459 / 60 / 3$ & $549 / 36 / 2$ & $0.063 / 0.034$ & $\begin{array}{l}1.87(\mathbf{1 . 2 6}-\mathbf{2 . 7 9}) / \\
\mathbf{1 . 9 9}(\mathbf{1 . 3 1}-\mathbf{3 . 0 4}) / \\
1.73(0.29-10.38)\end{array}$ & $\begin{array}{c}2.01 \\
(1.30-3.09)\end{array}$ & $\begin{array}{c}1.84 \\
(0.61-11.05)\end{array}$ & 0.004 \\
\hline & $\begin{array}{l}\text { Combined } \\
\text { set }\end{array}$ & $1083 / 186 / 6$ & $1464 / 172 / 8$ & $0.078 / 0.057$ & $\begin{array}{l}1.39(1.13-1.71) / \\
1.45(1.17-1.80) / \\
0.97(0.34-2.80)\end{array}$ & $\begin{array}{c}1.47 \\
(1.18-1.84)\end{array}$ & $\begin{array}{c}1.02 \\
(0.35-2.94)\end{array}$ & 0.002 \\
\hline
\end{tabular}

${ }^{\mathrm{a}} \mathrm{A} / \mathrm{G}$ represented major allele/minor allele, respectively.

${ }^{b}$ Genotypes were major homozygote/heterozygote/minor homozygote.

${ }^{\mathrm{c}} \mathrm{MAF}$, minor allele frequency in cancer-free controls.

${ }^{\mathrm{O}} \mathrm{OR}$, odds ratio; $\mathrm{CI}$, confidence interval. $P$ was for additive model. The logistic regression analysis was adjusted for age and sex. ehet, heterozygote vs. major homozygote; hom, minor homozygote vs. major homozygote.

Table 2: Subgroup analysis of clinical characteristics for the association between SNP rs4759314 and gastric cancer risk

\begin{tabular}{|c|c|c|c|c|c|}
\hline & $n$ & OR $(95 \% \text { CI })^{\mathrm{a}}$ & $\mathrm{AA}(n, \%)$ & AG/GG $(n, \%)$ & $P^{a}$ \\
\hline Controls & 1646 & Reference (1.00) & $1464(89.1)$ & $180(10.9)$ & \\
\hline \multicolumn{6}{|l|}{ Tumor site } \\
\hline Cardia & 403 & $1.58(1.16-2.15)$ & $339(84.1)$ & 64 (15.9) & 0.006 \\
\hline Non-cardia & 734 & $1.48(1.15-1.90)$ & $621(84.6)$ & $113(15.4)$ & 0.002 \\
\hline Both & 61 & $1.45(0.70-2.99)$ & $52(85.3)$ & $9(14.7)$ & 0.353 \\
\hline \multicolumn{6}{|c|}{ Histological type } \\
\hline Diffuse & 612 & $1.43(1.09-1.88)$ & $519(84.8)$ & $93(15.2)$ & 0.006 \\
\hline Intestinal & 513 & $1.56(1.17-2.07)$ & 433 (84.4) & $80(15.6)$ & 0.005 \\
\hline \multicolumn{6}{|l|}{ TNM stage } \\
\hline $\mathrm{I} / \mathrm{II}$ & 551 & $1.51(1.15-2.00)$ & $465(84.4)$ & $86(15.6)$ & 0.004 \\
\hline III/IV & 604 & $1.46(1.12-1.92)$ & $512(84.8)$ & $92(15.2)$ & 0.006 \\
\hline
\end{tabular}

${ }^{a} \mathrm{OR}$, odds ratio; CI, confidence interval. Adjusted for age and sex in logistic regression model.

Similar results were observed that $\mathrm{HOXC11}$ expression in $\mathrm{GC}$ cases with $\mathrm{AG}$ genotype was higher than those with AA genotype, although no statistical significance $(P=0.144$, Supplementary Figure 2a). Furthermore, we evaluated the correlation of these two genes in tumors, and found a positive correlation between HOTAIR and HOXC11 expression level $\left(\mathrm{R}^{2}=0.271, P<0.001\right.$, Figure 2c).

\section{Identification of promoter activity in SNP rs4759314 region}

Previous study has reported that HOTAIR intron 2 might perform enhancer activity for $\mathrm{H} 3 \mathrm{~K} 4 \mathrm{me} 1 / 3$ modification [16]. However, the HOTAIR intron 1, GC susceptibility SNP rs4759314 located, did not show any histone modification in this region (Supplementary 


\begin{tabular}{|c|c|c|}
\hline Study ID & OR $(95 \% \mathrm{Cl})$ & Weight \% \\
\hline Age & & \\
\hline$\leq 63$ & $1.23(0.91,1.67)$ & 26.02 \\
\hline$>63$ & $1.74(1.27,2.38)$ & 24.32 \\
\hline Subtotal $(I-$ squared $=58.7 \%, p=0.120)$ & $1.45(1.17,1.81)$ & 50.34 \\
\hline Sex & & \\
\hline Male & $1.63(1.24,2.13)$ & 32.77 \\
\hline Female & $1.17(0.80,1.70)$ & 16.88 \\
\hline Subtotal $(1-$ squared $=49.0 \%, p=0.161)$ & $1.46(1.17,1.81)$ & 49.66 \\
\hline Heterogeneity between groups: $p=0.994$ & & \\
\hline Overall $(I-$ squared $=31.6 \%, p=0.223$ ) & $1.46(1.25,1.70)$ & 100.00 \\
\hline $\begin{array}{l}1 \\
.42\end{array}$ & & \\
\hline
\end{tabular}

Figure 1: Subgroup analysis of demographic features for the association between SNP rs4759314 and gastric cancer risk in genetic dominant model. The red diamonds and blue horizontal lines correspond to the subgroup-specific OR and $95 \% \mathrm{CI}$; the orange diamonds represents the pooled OR with $95 \% \mathrm{CI}$.

Figure 1). Intriguingly, we identified that this region might produce promoter activity from ENCODE data; and we therefore examined the promoter activity of intron 1 region by luciferase reporter assays. As shown in Figure 2d, the luciferase activity of intron 1 region containing $\mathrm{G}$ allele was significantly higher compared to that of A allele in both MGC-803 and BGC-823 GC cells $(P=0.012$ and 0.010 , respectively), indicating that there might exist pre-transcriptional regulation, especially allele-specific modulation, on HOXC11 expression in region of HOTAIR intron 1, where some allele-specificbinding transcription factors are potentially involved in this region (Supplementary Table 3).

\section{Candidate genes expression level in GC tissues}

Considering several studies have demonstrated abnormal expression of HOTAIR in multiple tumors [4, $10,12,17]$, we then evaluated the expression of HOTAIR, as well as HOXC11, in GC tissues by using Oncomine database, in which datasets from Cho et al. [18] and D'Errico et al. [19] were selected for further analysis. As shown in Supplementary Figure 3a-3h, both HOTAIR and $\mathrm{HOXC11}$ expression levels in either GC diffuse or intestinal tissues were evidently higher than those in normal gastric tissues. Interestingly, we also observed a slight correlation between the expressions of HOTAIR and HOXC11 (Supplementary Figure 3i-3j). Moreover, the deregulation of $\mathrm{HOXC11}$ expression was supported by TCGA data, which showed dramatically higher expression of $\mathrm{HOXC11}$ in GC tissues than that in corresponding adjacent normal tissues $(P<0.001$, Supplementary Figure 2b).

\section{DISCUSSION}

In the present study, we performed a two-stage case-control study to evaluate the association between three SNPs in HOTAIR gene and GC risk. In our findings, SNP rs4759314 A > G was significantly associated with increased GC risk, especially in the elder and male subjects. In addition, the AG genotype was related to higher expression of HOTAIR and HOXC11 in GC tissues, compared to the AA genotype, for which the $\mathrm{G}$ allele enhanced higher promoter activity of HOXC11 than the A allele. This is the first study to assess the genetic role of HOTAIR genetic variants in GC susceptibility.

Emerging evidences have demonstrated that HOTAIR could act as an oncogene in multiple malignancies and its up-regulated expression may result in malignant transformation of normal cells [8]. Gupta et al. 
a.

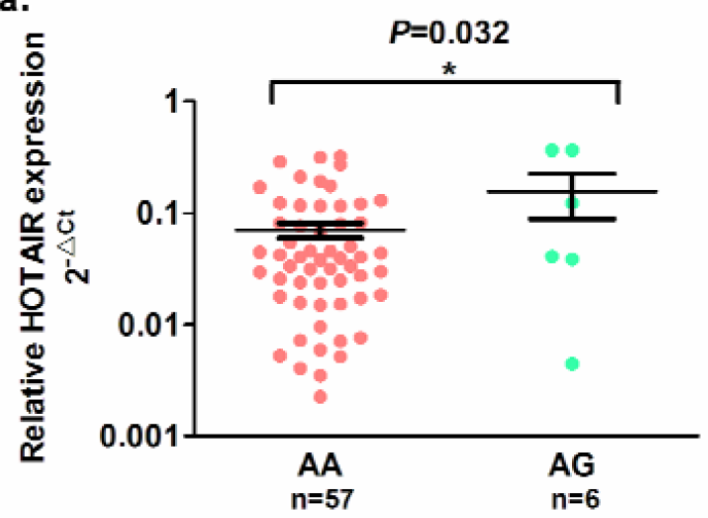

c.

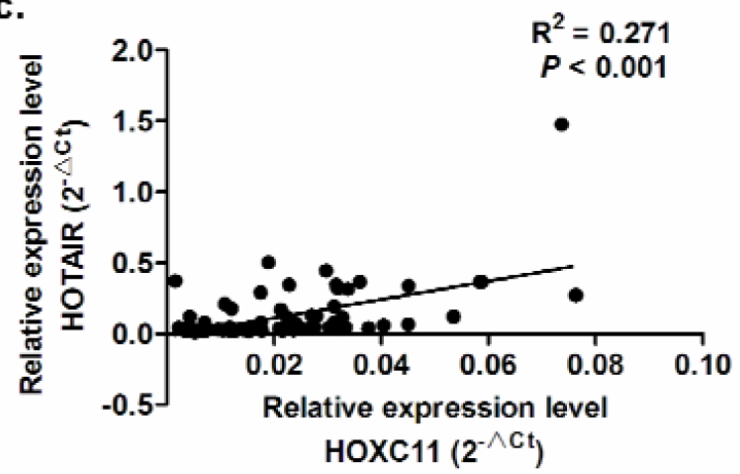

b.

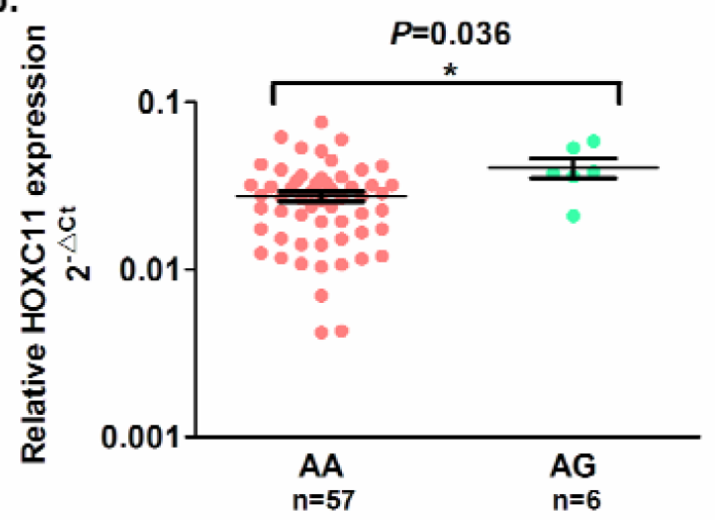

d.

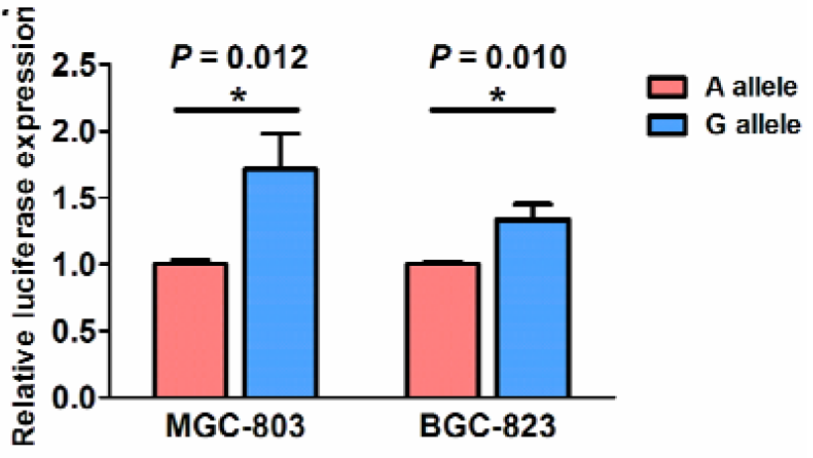

Figure 2: The allele-specific effects of SNP rs4759314 on HOTAIR and HOXC11 expression in 63 gastric cancer tissues and allele-specific promoter activity. The allele-specific effects showed significantly on HOTAIR $\mathbf{a}$. and HOXC11 b. The expression of HOTAIR and HOXC11 presented a slight correlation in gastric cancer tissues $\mathbf{c}$. expression level, as well on the intronic promoter activity d.

firstly identified the abnormal expression of HOTAIR in breast cancer tissues, which showed that high expression of HOTAIR in breast cancer tissues was associated with poor metastasis-free survival and overall survival [4]. Subsequently, similar phenomena have been identified in other solid tumors. For GC, the HOTAIR level was highly expressed and significantly associated with gastric cancer progresses and prognosis $[10,12,20,21]$. In this study, we identified the association of rs $4759314 \mathrm{G}$ allele and increased HOTAIR expression in GC tissues, which indicated biological rationality of this correlation.

Recently, several studies have investigated the genetic effects of HOATIR tagSNPs on cancer susceptibility. Zhang et al. initially identified 3 tagSNPs of HOTAIR gene (i.e., rs920778, rs1899663 and rs4759314) and evaluated their associations on esophageal squamous cell carcinoma (ESCC) risk; and they found that SNP rs920778 was dramatically related to increased ESCC risk and functional study demonstrated an allele-specific effect on the intronic enhancer activity of HOTAIR [16]. Subsequently, Guo et al. assessed the relationship between HOTAIR polymorphisms and gastric cardia adenocarcinoma (GCA) risk, and identified that tagSNP rs12826786 not only was associated with increased GCA risk, but also performed a genotype-specific effect on
HOTAIR expression [22]. In present study, although two of three tagSNPs were not the same as reported tagSNPs, they were in the same block region [15] and showed high correlation (1.0 of $\mathrm{r}^{2}$ between rs7958904 and rs920778; 1.0 for rs874945 and rs12826786; Supplementary Table 4). Given these, our identified risk SNP rs4759314 in GC susceptibility was quite different from previously reported risk SNPs. Additionally, in our previous study, we observed a significant association between the SNP rs7958904 and decreased risk of colorectal cancer and found an allele-specific phenotype of SNP rs7958904 in colorectal cancer cell proliferation [15]. All of these indicated an important role of HOTAIR genetic variants in cancer susceptibility.

Considering that recognizing the precise targets of risk variants located in noncoding regions was still the major challenge [23], we hypothesized that the risk SNP rs4759314 falling in intron of HOTAIR gene had a genotype-phenotype correlation on its nearby genes. Intriguingly, we observed that the intron region of SNP rs4759314 resided performed a promoter activity from ENCODE data, and gene HOXC11 was only located in about 5-kb downstream of SNP rs4759314, which was involved in the development and outcome of cancer [24]. We hypothesized whether the intronic region of 
SNP rs4759314 resided existed allele-specific mediated promoter activity [25]. Thus, we explored the potential transcription factors on this candidate intron of HOTAIR by two online bioinformatics tools, from which some conventional transcription factors were predicted and also were affected by SNP rs4759314 alleles. This phenotype was validated in further functional experiments: the luciferase activity of risk allele G of rs4759314 was higher than that of A allele, and SNP rs4759314 presented an genotype-specific expression on $\mathrm{HOXC11}$ expression. Besides, this phenomenon was supported by TCGA database, although no statistical significance in genotype-specific expression analysis, which might be for genetic ancestry of European populations (minor allele frequency $=0.017$ of SNP rs $4759314 \mathrm{G}$ allele from CEU populations of 1000 Genomics Project) is different from that of Asians ( 0.100 of G allele in CHB and JPT populations).

In conclusion, we identified that a functional SNP rs4759314 of HOTAIR had a strong association with GC susceptibility in the Chinese populations. SNP rs4759314 resided in an intronic promoter region influenced the activity of this promoter, contributing to a genotypespecific effect on the expression of host gene HOTAIR and its downstream gene $\mathrm{HOXC11}$, which is a potential mechanism for GC susceptibility. All these indicated that HOTAIR and its genetic variations may be a potential biomarker for risk assessment, early detection and therapeutic target of gastric cancer; and larger prospective and experimental studies were warranted to further validation.

\section{MATERIALS AND METHODS}

\section{Study subjects}

This study was approved by the Institutional Review Board of Nanjing Medical University. All subjects enrolled were heritably unrelated ethnic Han Chinese. The patients were recruited in our ongoing GC study, in which individuals were from the Second Affiliated Hospital of Nanjing Medical University, Cancer Hospital of Nantong and Yixing Cancer Hospital from March 2006 to January 2011 which described in detail previously [26, 27]. Briefly, a total of $1,275 \mathrm{GC}$ cases and 1,646 cancer-free controls were recruited in two stages ( 753 cases and 1,057 controls for the test set, with additional 522 cases and 589 controls for the validation set). The controls frequencymatched to cases on age ( \pm 5 years) and sex were randomly enrolled in the same hospital for a routine examination of physical conditions. All the patients were histologically confirmed and the clinical information was obtained, including tumor site (cardia/non-cardia/both), histological type and clinical tumor node metastasis (by UICC/AJCC criteria of TNM stage).

\section{SNP selection and genotyping}

In our previous study, we have identified three tagSNPs of HOTAIR gene (i.e., rs4759314, rs7958904 and rs874945) [15]. All samples were genotyped by TaqMan allelic discrimination methods with ABI 7900HT real-time PCR system (Applied Biosystems, Foster City, CA, USA). The average call rates for each SNP were over $98 \%$; the random $10 \%$ of samples were repeatedly genotyped and the concordance rate was $100 \%$.

\section{Analysis of HOTAIR and HOXC11 expression in gastric cancer tissues}

A total of $63 \mathrm{GC}$ tissues were obtained from pretreatment patients. Total RNA were extracted from tissues by using TRIzol reagent (Invitrogen, Carlsbad, CA, USA) according to the protocol. The cDNA was then synthesized with M-MLV reverse transcriptase (Invitrogen). Subsequently, the expression of HOTAIR and $\mathrm{HOXC11}$ were determined by SYBR Green Assay (TaKaRa Biotechnology, Dalian, China) and the levels were normalized by Glyceraldehyde 3-phosphate dehydrogenase (GAPDH) by the $2^{-\triangle \mathrm{Ct}}$ method. All assays were conducted by using the ABI 7900 HT real-time PCR system (Applied Biosystems, Foster City, CA, USA). All reactions were performed in triplicate.

\section{Detecting promoter activity of SNP rs4759314}

The luciferase reporter assay was used to detect the effect of SNP rs4759314 on HOTAIR promoter activity. The sequences of target intron with SNP rs4759314 A or $\mathrm{G}$ alleles were gene-synthesized and then were cloned into Xho 1/Hind III restrictive sites of pGL3enhancer plasmid (Promega, Madison, WI, USA), and the accuracy was confirmed by DNA sequencing. MGC-803 and BGC-823 cells were cultured in 24-well plates and transfected with $800 \mathrm{ng}$ of each allele-specific plasmids using Lipofectamine ${ }^{\mathrm{TM}} 2000$ (Invitrogen, Carlsbad, CA, USA), respectively. The pRL-SV40 (Promega, Madison, WI, USA) as internal control was cotransfected into cells. After $48 \mathrm{~h}$ transfection, dual luciferase activities were determined using a Luciferase Reporter Assay System (Promega, Madison, WI, USA). All assays were conducted in independent triplicate.

\section{Statistical analysis}

Differences in the distribution of selected demographic variables and genotypes of tagSNPs were evaluated by Pearson's $\chi^{2}$ test. Hardy-Weinberg equilibrium (HWE) for each SNP among controls was tested using a goodness-of-fit $\chi^{2}$-test. The associations of each SNP and GC susceptibility were estimated by using unconditional logistic regression analyses with odds ratios 
(ORs) and 95\% confidence intervals (CIs). Variables of age and sex were as covariates adjusted for the association analysis. Multiple genetic models (i.e., additive, dominant, recessive and co-dominant models) were applied to assess the significance of SNPs. In additional subgroup analysis, the heterogeneity was evaluated by the $\chi^{2}$ based on $Q$-test. Differences in allele-specific promoter activity and gene expression were compared by Student's $t$-test or paired $t$-test. A $P$ value of less 0.05 for two-side was considered statistically significant. All analyses were conducted with SAS 9.1.3 software (SAS Institute, Inc., Cary, NC, USA).

\section{ACKNOWLEDGMENTS}

This study was partly supported by National Natural Science Foundation of China (81473049 and 81230068), the Key Program for Basic Research of Jiangsu Provincial Department of Education (12KJA330002), Jiangsu Provincial Science and Technology Innovation Team, Collaborative Innovation Center For Cancer Personalized Medicine, and the Priority Academic Program Development of Jiangsu Higher Education Institutions (Public Health and Preventive Medicine).

\section{CONFLICTS OF INTEREST}

We declared no conflicts of interests in this study.

\section{REFERENCES}

1. Ferlay J, Soerjomataram I, Dikshit R, Eser S, Mathers C, Rebelo M, Parkin DM, Forman D, Bray F. Cancer incidence and mortality worldwide: Sources, methods and major patterns in GLOBOCAN 2012. Int J Cancer. 2015; 136:E359-386.

2. Jemal A, Bray F, Center MM, Ferlay J, Ward E, Forman D. Global cancer statistics. CA Cancer J Clin. 2011; 61:69-90.

3. Moran VA, Perera RJ, Khalil AM. Emerging functional and mechanistic paradigms of mammalian long non-coding RNAs. Nucleic Acids Res. 2012; 40:6391-6400.

4. Gupta RA, Shah N, Wang KC, Kim J, Horlings HM, Wong DJ, Tsai MC, Hung T, Argani P, Rinn JL, Wang Y, Brzoska P, Kong B, et al. Long non-coding RNA HOTAIR reprograms chromatin state to promote cancer metastasis. Nature. 2010; 464:1071-1076.

5. Zhang H, Chen Z, Wang X, Huang Z, He Z, Chen Y. Long non-coding RNA: a new player in cancer. J Hematol Oncol. 2013; 6:37.

6. Gutschner T, Diederichs S. The hallmarks of cancer: a long non-coding RNA point of view. RNA Biol. 2012; 9:703-719.

7. Padua Alves C, Fonseca AS, Muys BR, de Barros ELBR, Burger MC, de Souza JE, Valente V, Zago MA,
Silva WA Jr. Brief report: The lincRNA Hotair is required for epithelial-to-mesenchymal transition and stemness maintenance of cancer cell lines. Stem Cells. 2013; 31:2827-2832.

8. Cai B, Song XQ, Cai JP, Zhang S. HOTAIR: a cancer-related long non-coding RNA. Neoplasma. 2014; 61:379-391.

9. Kim K, Jutooru I, Chadalapaka G, Johnson G, Frank J, Burghardt R, Kim S, Safe S. HOTAIR is a negative prognostic factor and exhibits pro-oncogenic activity in pancreatic cancer. Oncogene. 2013; 32:1616-1625.

10. Xu ZY, Yu QM, Du YA, Yang LT, Dong RZ, Huang L, Yu PF, Cheng XD. Knockdown of long non-coding RNA HOTAIR suppresses tumor invasion and reverses epithelial-mesenchymal transition in gastric cancer. Int J Biol Sci. 2013; 9:587-597.

11. Endo H, Shiroki T, Nakagawa T, Yokoyama M, Tamai K, Yamanami H, Fujiya T, Sato I, Yamaguchi K, Tanaka N, Iijima K, Shimosegawa T, Sugamura K, et al. Enhanced expression of long non-coding RNA HOTAIR is associated with the development of gastric cancer. PLoS One. 2013; 8:e77070.

12. Liu XH, Sun M, Nie FQ, Ge YB, Zhang EB, Yin DD, Kong R, Xia R, Lu KH, Li JH, De W, Wang KM, Wang ZX. Lnc RNA HOTAIR functions as a competing endogenous RNA to regulate HER 2 expression by sponging miR-331-3p in gastric cancer. Mol Cancer. 2014; 13:92.

13. Verhaegh GW, Verkleij L, Vermeulen SH, den Heijer M, Witjes JA, Kiemeney LA. Polymorphisms in the H19 gene and the risk of bladder cancer. Eur Urol. 2008; 54:1118-1126.

14. Xue Y, Wang $M$, Kang M, Wang Q, Wu B, Chu H, Zhong D, Qin C, Yin C, Zhang Z, Wu D. Association between lncrna PCGEM1 polymorphisms and prostate cancer risk. Prostate Cancer Prostatic Dis. 2013; 16:139-144. S131.

15. Xue Y, Gu D, Ma G, Zhu L, Hua Q, Chu H, Tong N, Chen J, Zhang Z, Wang M. Genetic variants in lncRNA HOTAIR are associated with risk of colorectal cancer. Mutagenesis. 2015; 30:303-10.

16. Zhang X, Zhou L, Fu G, Sun F, Shi J, Wei J, Lu C, Zhou C, Yuan Q, Yang M. The identification of an ESCC susceptibility SNP rs920778 that regulates the expression of lncRNA HOTAIR via a novel intronic enhancer. Carcinogenesis. 2014; 35:2062-2067.

17. Niinuma T, Suzuki H, Nojima M, Nosho K, Yamamoto H, Takamaru H, Yamamoto E, Maruyama R, Nobuoka T, Miyazaki Y, Nishida T, Bamba T, Kanda $T$, et al. Upregulation of miR-196a and HOTAIR drive malignant character in gastrointestinal stromal tumors. Cancer Res. 2012; 72:1126-1136.

18. Cho JY, Lim JY, Cheong JH, Park YY, Yoon SL, Kim SM, Kim SB, Kim H, Hong SW, Park YN, Noh SH, Park ES, Chu IS, et al. Gene expression signature-based 
prognostic risk score in gastric cancer. Clin Cancer Res. 2011; 17:1850-1857.

19. D’Errico M, de Rinaldis E, Blasi MF, Viti V, Falchetti M, Calcagnile A, Sera F, Saieva C, Ottini L, Palli D, Palombo F, Giuliani A, Dogliotti E. Genome-wide expression profile of sporadic gastric cancers with microsatellite instability. Eur J Cancer. 2009; 45:461-469.

20. Hajjari M, Behmanesh M, Sadeghizadeh M, Zeinoddini M. Up-regulation of HOTAIR long non-coding RNA in human gastric adenocarcinoma tissues. Med Oncol. 2013; 30:670.

21. Okugawa Y, Toiyama Y, Hur K, Toden S, Saigusa S, Tanaka K, Inoue Y, Mohri Y, Kusunoki M, Boland CR, Goel A. Metastasis-associated long non-coding RNA drives gastric cancer development and promotes peritoneal metastasis. Carcinogenesis. 2014; 35:2731-2739.

22. Guo W, Dong Z, Bai Y, Guo Y, Shen S, Kuang G, Xu J. Associations between polymorphisms of HOTAIR and risk of gastric cardia adenocarcinoma in a population of north China. Tumour Biol. 2015; 36:2845-54.

23. Edwards SL, Beesley J, French JD, Dunning AM. Beyond GWASs:illuminating the dark road from association to function. Am J Hum Genet. 2013; 93:779-797.
24. Liu YJ, Zhu Y, Yuan HX, Zhang JP, Guo JM, Lin ZM. Overexpression of HOXC11 homeobox gene in clear cell renal cell carcinoma induces cellular proliferation and is associated with poor prognosis. Tumour Biol. 2015; $36: 2821-2829$.

25. Huang Q, Whitington T, Gao P, Lindberg JF, Yang Y, Sun J, Vaisanen MR, Szulkin R, Annala M, Yan J, Egevad LA, Zhang K, Lin R, et al. A prostate cancer susceptibility allele at $6 \mathrm{q} 22$ increases RFX6 expression by modulating HOXB13 chromatin binding. Nat Genet. 2014; 46:126-135.

26. Song P, Zhu H, Zhang D, Chu H, Wu D, Kang M, Wang M, Gong W, Zhou J, Zhang Z, Zhao Q. A genetic variant of miR-148a binding site in the SCRN1 3'-UTR is associated with susceptibility and prognosis of gastric cancer. Sci Rep. 2014; 4:7080.

27. Xu M, Qiang F, Gao Y, Kang M, Wang M, Tao G, Gong W, Zhu H, Wu D, Zhang Z, Zhao Q. Evaluation of a Novel Functional Single-Nucleotide Polymorphism (rs35010275 G > C) in MIR196A2 Promoter Region as a Risk Factor of Gastric Cancer in a Chinese Population. Medicine (Baltimore). 2014; 93:e173. 